\title{
Opinião/Opinion/Opinión
}

Revista Brasileira de Extensão Universitária

v. 5, n. 2, p. 69-75, jul.- dez. 2014

e-ISSN 2358-0399

originais recebidos em 01 de janeiro de 2015

aceito para publicação em 06 de março de 2015

Fórum de

Pró-Reitores

de Extensão

das Instituições

Públicas de

Educação Superior

Brasileiras

\section{Revistas acadêmicas de extensão universitária no Brasil}

\author{
Geraldo Ceni Coelho *
}

\begin{abstract}
Resumo: A publicação de revistas multidisciplinares dedicadas à extensão universitária é um fenômeno notável em vários países da América. Os periódicos mais antigos desta natureza são dos anos 1960, nos Estados Unidos, quando surgiu o Journal of Extension. No Brasil, foram detectadas 29 revistas multidisciplinares dedicadas à extensão universitária ainda em atividade, e as mais antigas entre elas foram iniciadas nos anos 1990. Porém, outras revistas com perfil editorial semelhantes foram encontradas e, embora descontinuadas, iniciaram na década anterior. A região Sul apresenta 12 (41\%) das revistas em atividade, e a região sudeste nove (31\%), o que indica uma distribuição geográfica desigual no país. O número de revistas multidisciplinares dedicadas à extensão universitária cresceu muito, praticamente triplicando nos últimos dez anos. Nas revistas brasileiras, há uma forte predominância de trabalhos publicados cujo conteúdo se refere a relatos de experiências de projetos de extensão. Poucos são os trabalhos oriundos da pesquisa na extensão universitária, ou em temas que possam subsidiá-la. A qualidade editorial da maioria das revistas apresenta aspectos a serem aperfeiçoados, destacando-se, o corpo editorial e a periodicidade.
\end{abstract}

Palavras-chave: edição eletrônica, extensão universitária, periódicos.

\section{Academic Journals on University Extension in Brazil}

Abstract: The publication of multidisciplinary journals dedicated to university extension is a remarkable phenomenon in several countries in the American continent. The oldest publications of this nature date back to the 1960s in the United States, when the first issue of the Journal of Extension was released. In Brazil, 29 multidisciplinary active journals dedicated to university extension can be detected, being the oldest among them released in the 1990s. Other journals with similar editorial profile were found in the previous decade, however, these publications were later discontinued. In the Southern region of Brazil there are $12(41 \%)$ of the extension journals in activity, and in the Southeast region there are nine (31\%), indicating an uneven geographical distribution in the country. The number of multidisciplinary journals dedicated to university extension has almost tripled over the last ten years. In the Brazilian journals, there is a strong predominance of publications whose content regarding experience reports of extension projects. A few are derived from research activities in university extension projects, or on topics that may relate to it. The editorial quality of most journals shows areas to be potentially improved, especially regarding the editorial board and the periodicity.

Keywords: electronic edition, university extension, periodicals. 


\section{Las revistas académicas de extensión universitaria en Brasil}

Resumen: La publicación de revistas multidisciplinares dedicadas a la extensión universitaria es un fenómeno notable en muchos países de América. Las revistas más antiguas de esta naturaleza son de los años 60, en los Estados Unidos, cuando surgió el Journal of Extension. En Brasil, se detectaron 29 revistas multidisciplinares dedicadas a la extensión universitaria y que siguen activas, siendo las primeras iniciadas en la década de 1990. Sin embargo, se verificaron otras revistas con perfil editorial similar y, aunque discontinuadas, se iniciaron en la década anterior. La región sur de Brasil tiene $12(41 \%)$ de las revistas de extensión en actividad, y la región sudeste tiene nueve (31\%), lo que indica una distribución geográfica desigual en el país. El número de revistas multidisciplinares dedicadas a la extensión universitaria ha crecido hasta casi el triple en los últimos diez años. En las revistas brasileñas, hay un fuerte predominio de artículos cuyo contenido se refiere a informes de experiencias de proyectos de extensión. Son pocos los trabajos son derivados de la investigación en la extensión universitaria, o en temas que puedan subsidiarla. La calidad editorial de la mayoría de las revistas muestra áreas de mejora potencial, destacando el consejo editorial y la periodicidad.

Palabras-clave: edición electrónica, extensión universitaria, publicaciones periódicas.

\section{Introdução}

Tornar público, por escrito, opiniões, teorias e ideias, é uma marca da civilização. A partir do surgimento da ciência, entre os séculos XVI e XVII, a publicação das investigações e reflexões por meio de livros, ganhou papel essencial para o reconhecimento das conquistas intelectuais de seus autores. O formato de comunicações curtas em revistas especializadas, hoje conhecidos como periódicos, tomou forma no final do século XVII. O primeiro periódico acadêmico, similar aos moldes atuais, foi o Le Journal des Sçavans (atualmente Journal des Savants), no ano de 1665, na França. No mesmo ano, iniciou-se a publicação do periódico Philosophical Transactions (mais tarde com o nome Philosophical Transactions of the Royal Society), vinculado à Real Sociedade de Londres, reconhecida como a primeira sociedade científica (BROWN, 1972; WIKIPEDIA, 2015). Desde então, o número de periódicos científicos tem crescido a uma taxa de aproximadamente $3 \%$ ao ano (JINHA, 2010).

Publicar tem função de dar créditos e reconhecimento aos autores e, ao mesmo tempo, contribuir para o enriquecimento da cultura científica enquanto patrimônio da humanidade. Especificamente, serve para guiar os passos da academia e da sociedade em geral na sua busca por novos conhecimentos, evitando que o esforço técnico e científico tenha que ser repetido.

A publicação acadêmica tem sido primordialmente vinculada à atividade de pesquisa, ou seja, destinada a divulgar o produto das atividades de investigação ou reflexão teórica, dentro das mais diversas áreas de conhecimento.

A extensão, enquanto função universitária formalmente reconhecida, surgiu apenas no final do século XIX (SOUSA, 2010; COELHO, 2014). Porém, a publicação advinda da extensão vinculada a periódicos acadêmicos é bem mais recente. Nos Estados Unidos, o periódico multidisciplinar mais antigo, especializado em divulgação da extensão universitária, é o Journal of Extension, iniciado em 1963. No Brasil, as revistas mais antigas dedicadas à extensão universitária em um conceito mais amplo (multidisciplinares ${ }^{1}$ ), e ainda em atividade, são a revista Participação (1997) e a revista Em Extensão (1998), vinculadas à UnB e à UFU, respectivamente. Porém, cabe ressaltar que o periódico Cadernos da Pró-Reitoria de Extensão da PUC Minas (ISSN 0104-4397) iniciou suas atividades em 1988, encerrando em 2006. A Revista Desafio da Universidade Federal do Ceará também iniciou sua publicação em 1988, circulando até a década seguinte. Por sua vez, a Revista de Extensão da UFPB (ISSN 1414-6495) foi ativa entre 1996 e 1999.

\section{$O$ que se publica nos periódicos de extensão universitária}

As publicações vinculadas à pesquisa são comunicações de resultados de investigações, opiniões, revisões ou meta-análises, e artigos teóricos. Se a pesquisa é geradora de novos conhecimentos que devem ser comunicados, o que se publica a partir da extensão? Analisando as publicações veiculadas pelas revistas dedicadas à extensão (listadas nas Tabelas 1 e 2 ), percebe-se que as produções são classificadas de diferentes formas, e não há um conceito unívoco sobre a definição de categorias. Desta forma, segue-se uma tentativa de delimitá-las:

a. Artigos teóricos e de opinião: artigos que buscam descrever ideias, propostas e conceitos sobre extensão universitária de uma forma ampla. Não se vinculam especificamente a projetos, programas, ou resultados específicos de atividades de extensão;

b. Relatos de experiência: descrição das atividades e ações de extensão e seus resultados, de forma crítica e reflexiva, eventualmente apresentando dados quantitativos relacionados à execução destas ações de extensão; 
c. Artigos de pesquisa: comunicações de resultados de investigação sobre a extensão universitária enquanto objeto de estudo ou investigações sobre temas relevantes aplicados ou estreitamente relacionados com a extensão. Muitas vezes, tratam de pesquisas sobre o impacto da extensão universitária sobre a qualidade de ensino, a formação acadêmica, a sociedade extramuros e sobre a própria comunidade acadêmica em seus diferentes segmentos.

Algumas vezes, porém, artigos de pesquisa sobre outros temas, com alguma vinculação a ações de extensão (ou mesmo nenhuma vinculação explicitamente evocada) são também publicados, o que pode indicar uma falta de delimitação editorial mais precisa do escopo do periódico.

Os relatos de experiência contribuem para inspirar e guiar novas propostas, chamando a atenção para entraves, desafios e oportunidades de intervenções extensionistas. Mais ainda, aspectos metodológicos utilizados em programas já executados servem como subsídio para novas proposições. Infelizmente, no campo editorial brasileiro a análise crítica das experiências extensionistas é pouco explorada. Muitas vezes, os relatos de extensão são entendidos como uma forma de divulgação pura e simples das ações de extensão, tendo assim uma intenção ou conteúdo de relatório.

Os relatos de experiência são a grande maioria das publicações nas revistas de extensão no Brasil, seguidos pelos artigos teóricos. São raros os artigos de pesquisa tendo como tema a extensão ou abordagens de investigação estritamente relacionadas à extensão. Já nos Estados Unidos, observa-se uma outra proporção entre as categorias de publicação. Tomando-se o exemplo do periódico Journal of Extension, observa-se no volume 52 (ano de 2014) que os relatos de experiência (seções 'Ideas at Work' e 'Tools of Trade') representam cerca de $50 \%$ do número de artigos publicados. Os artigos teóricos perfazem $10 \%$ e os artigos de pesquisa $40 \%$, aproximadamente.

\section{As revistas de extensão universitária no Brasil}

Os periódicos dedicados à extensão universitária no Brasil que estão em atividade e publicaram pelo menos uma edição durante o ano de 2014 (até dezembro de 2014) são em número de 29 (Tabela 1 e Figura 1$)^{2}$. Destes, 25 possuem edição eletrônica (Tabela 1). Percebe-se uma distribuição geográfica desigual das editoras das revistas de extensão universitária em atividade: 12 estão na região sul, nove na região sudeste, cinco na região nordeste, duas no distrito federal e uma na região norte. O Rio Grande do Sul e Santa Catarina são estados que se destacam quanto ao número de revistas em atividade, com cinco revistas cada um (Figura 1).
O número de periódicos brasileiros dedicados à extensão universitária é algo notável, visto que o número de periódicos semelhantes em outros países da América Latina e Estados Unidos são oito ${ }^{3}$ (Tabela 2). Mais ainda, o número de revistas de extensão universitária tem crescido expressivamente. Há dez anos eram nove em atividade e há cinco anos, já somavam 18 (Figura 2). Por outro lado, existe um número expressivo de revistas inativas $^{4}$. Entre os anos 1988 e 2014 foram criadas outras 17 revistas de extensão universitária que descontinuaram sua atividade de publicação (dados não mostrados).

\section{Sobre a qualidade editorial}

São inúmeros os aspectos considerados na avaliação de qualidade de periódicos acadêmicos (KRZYZANOWSKI; FERREIRA, 1998, LIMA; MIRANDA, 2011) e considerar sua totalidade vai além do objetivo do presente artigo. Alguns aspectos são apresentados a seguir.

\section{ISSN eletrônico}

Entre os 29 periódicos em atividade no Brasil (Tabela 1), 25 possuem edição eletrônica, 24 utilizam a plataforma SEER (OJS) e 23 possuem ISSN eletrônico exclusivo. Porém, duas, utilizam o mesmo ISSN para a versão eletrônica e impressa. O ISSN eletrônico é fundamental para a recuperação do periódico na rede mundial de computadores, permitindo o cadastro em base de dados internacionais. Quando um periódico é divulgado em meios diferentes, impresso e digital, recomenda-se o uso de ISSN distintos para cada formato (LIMA; MIRANDA, 2011).

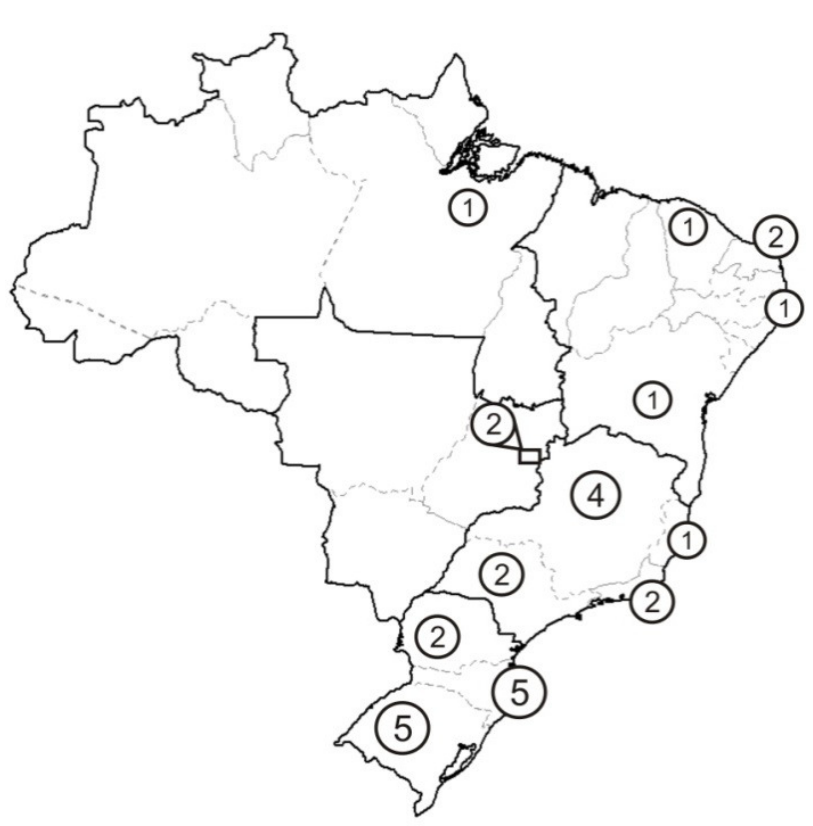

Figura 1. Distribuição geográfica dos periódicos de extensão universitária multidisciplinares no Brasil, tomando em consideração a localização da instituição editora. 
Tabela 1. ISSN, nome dos periódicos dedicados à extensão universitária no Brasil que estão em atividade e publicaram pelo menos uma edição durante o ano de 2014 (até fevereiro de 2015), instituição editora e ano da primeira publicação.

\begin{tabular}{|c|c|c|c|}
\hline ISSN & Título & Instituição Editora & Início \\
\hline $2238-6963$ & $\underline{\text { ParticipAção }}$ & UnB & 1997 \\
\hline $1982-7687$ & $\underline{\text { Em Extensão }}$ & UFU & 1999 \\
\hline $2358-8195$ & $\underline{\text { Expressa Extensão }}$ & UFPel & 1999 \\
\hline $1677-8898$ & $\underline{\text { Dialogos }}$ & $\mathrm{UCB}$ & 2002 \\
\hline $1806-2695$ & $\underline{\text { Revista Bras. de Extensão Universitária }}$ & FORPROEX/UFFS & 2003 \\
\hline $1807-0221$ & $\underline{\text { Extensio - Revista Eletrônica de Extensão }}$ & UFSC & 2004 \\
\hline $1679-4605$ & $\underline{\text { Revista Ciência e Extensão }}$ & UNESP & 2004 \\
\hline $2238-7315$ & $\underline{\text { Revista Conexão UEPG }}$ & UEPG & 2005 \\
\hline $1809-1636$ & Vivências: Ver. Eletrônica de Extensão da URI & URI-RS & 2005 \\
\hline $2236-4447$ & $\underline{\text { Interagir: Pensando a Extensão }}$ & UERJ & 2006 \\
\hline $1982-7776$ & $\underline{\text { UDESC em Ação }}$ & UDESC & 2007 \\
\hline $2358-7180$ & $\underline{\text { Extensão em Foco }}$ & UFPR & 2008 \\
\hline $2316-9060$ & $\underline{\text { Revista de Cultura e Extensão }}$ & USP & 2009 \\
\hline 2238-0167 (i.)* & $\underline{\text { Revista da Extensão }}$ & UFRGS & 2009 \\
\hline $2176-4867$ & $\underline{\text { Cataventos }}$ & UNICRUZ & 2009 \\
\hline $2178-6054$ & $\underline{\text { Revista Extensão e Sociedade }}$ & UFRN & 2010 \\
\hline $2236-6784$ & $\underline{\text { Revista Extensão }}$ & UFRB & 2011 \\
\hline $2316-400 X$ & $\underline{\text { Revista Extensão em Ação }}$ & UFC & 2011 \\
\hline $2317-5451$ & $\underline{\text { Elo- Diálogos em Extensão }}$ & UFV & 2012 \\
\hline $2318-3640$ & $\underline{\text { Extramuros }}$ & UNIVASF & 2013 \\
\hline $2317-7705$ & $\underline{\text { Raízes e Rumos }}$ & UNIRIO & 2013 \\
\hline $2317-8116$ & $\underline{\text { Revista Universo \& Extensão }}$ & UFPA & 2009 \\
\hline $2318-2326$ & $\underline{\text { Interfaces }}$ & UFMG & 2013 \\
\hline $2318-2350$ & $\underline{\text { Extendere }}$ & UERN & 2013 \\
\hline $2318-2539$ (i.)* & Extensão \& Comunidade & CEFET-MG & 2013 \\
\hline $2318-9665$ & $\underline{\text { Viver IFRS }}$ & IFRS & 2013 \\
\hline 2359-0599 & $\underline{\text { Caminho Aberto }}$ & IFSC & 2014 \\
\hline 2358-2499 (i.)* & Extensão Tecnológica & IFSC & 2014 \\
\hline 2316-2007 (i.)** & $\underline{\text { Guará - Revista de Extensão }}$ & UFES & 2014 \\
\hline
\end{tabular}

* (i.) = ISSN da versão impressa, não possui e-ISSN; * ** não publicou edição eletrônica.

Fonte: elaboração do autor.

\section{Abertura do corpo editorial}

O corpo editorial evidencia a autoridade acadêmica associada ao periódico. Supõe-se uma formação qualificada e uma interação entre os membros (LIMA; MIRANDA, 2011). O LATINDEX, serviço de indexação da Universidade Autônoma do México - UNAM, considera a abertura editorial entre os 36 quesitos de avaliação dos periódicos indexados em sua base. Considera-se uma abertura editorial satisfatória quando 2/3 dos membros do corpo editorial são de outras instituições, diferentes da instituição editora do 
periódico. Tendo em conta este parâmetro, observa-se que somente cinco (17\%) dos periódicos de extensão universitária em atividade alcançam esta condição. De outra parte, os termos utilizados para a definição de corpo editorial são bastante variáveis: conselho editorial, comitê científico, equipe de editores, etc. Muitas vezes, um periódico possui, ao mesmo tempo, diferentes instâncias que poderiam estar exercendo a função do conselho editorial, por exemplo, conselho científico e comitê editorial.

\section{Periodicidade e número de artigos}

A periodicidade e a regularidade na publicação são também aspectos da qualidade dos periódicos. Alguns autores consideram que a periodicidade quadrimestral é a freqüência mínima de publicação (LIMA; MIRANDA, 2011), e este também é o critério atual da base SciELO (SciELO, 2014).

Tabela 2. ISSN, nome dos periódicos dedicados à extensão universitária em países da América em atividade, instituição editora e ano da primeira publicação.

\begin{tabular}{llll}
\hline ISSN & Nome da Revista & País & Início \\
\hline $1852-9569$ & Extensión en Red & Argentina & 2010 \\
\hline $2250-4591$ & + E / Revista de Extensión Universitaria & Argentina & 2011 \\
$2250-7272$ & ExT: Revista de Extensión de la UNC & Argentina & 2012 \\
\hline--- & Revista Identidad y Territorio - ULEU & Argentina* & 2012 \\
\hline $1077-5315$ & Journal of Extension & Estados Unidos & 1963 \\
$1994-0219$ & Michigan Journal of Community Service Learning & Estados Unidos & 1994 \\
\hline $1534-6104$ & Journal of Higher Education Outreach and Engagement & Estados Unidos & 1996 \\
\hline $1555-9033$ & $\begin{array}{l}\text { International Journal for Service Learning in Engineering, } \\
\text { Humanitarian Engineering and Social Entrepreneurship }\end{array}$ & Estados Unidos & 2006 \\
\hline
\end{tabular}

* A Revista Identidad y Territorio é vinculada à Union Latinoamericana de Extensión Universitaria, cuja direção é itinerante entre os países membros, portanto a localização indicada aqui é temporária. Fonte: elaboração do autor.

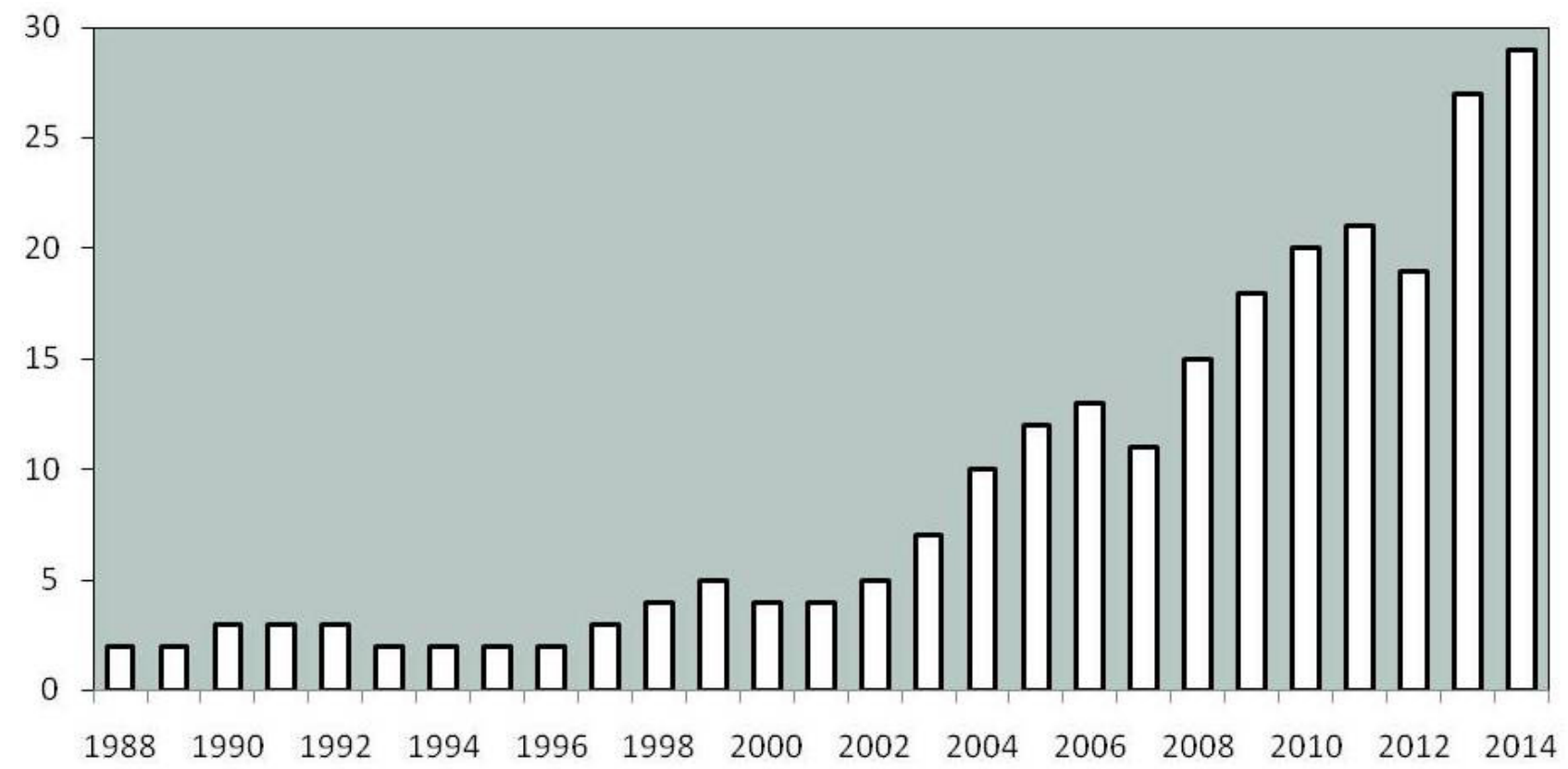

Figura 2. Número de periódicos de extensão universitária multidisciplinares em atividade no Brasil, entre os anos de 1998 e 2014. Inclui as revistas que cessaram suas atividades. 
Das 29 revistas de extensão analisadas (Tabela 1), uma mantém periodicidade quadrimestral, 18 mantém periodicidade semestral e 10 revistas são anuais ${ }^{5}$. Portanto, 68\% das revistas publicam pelo menos dois números por ano. Porém, algumas revistas não conseguem manter sua regularidade, o que na prática implica em uma periodicidade menor, e compromete a credibilidade editorial.

Analisando uma amostra de revistas $(n=16)^{6}$ no quesito número de artigos publicados anualmente entre 2013 e 2014, verifica-se uma média anual de 19,3 $\pm 12,7$. Apenas cinco destas revistas apresentam um número igual ou superior a 25 artigos por ano, número considerado recomendável pelo SciELO (2014) como valor mínimo na área de Ciências Humanas. O elevado desvio-padrão mostra grande dissimilaridade e amplitude entre as revistas, neste aspecto.

\section{Considerações finais}

A publicação em periódicos multidisciplinares dedicados à extensão universitária é bastante expressiva no Brasil, destacando-se em relação a outros países. O número de periódicos tem crescido bastante nos últimos anos. Resta saber se a produção intelectual voltada à extensão universitária terá fôlego para alimentar tantos periódicos, tanto em quantidade de artigos quanto em qualidade. Observa-se que são muitas as revistas que interromperam suas atividades nos últimos dez anos, o que indica a dificuldade da manutenção das atividades editoriais.

A produção intelectual de artigos nas revistas de extensão possivelmente tem uma correlação estreita com o financiamento público à extensão universitária, especialmente àquelas ações que não visam retorno financeiro. Portanto, a continuidade das políticas de apoio e financiamento, aliada às políticas públicas voltadas às questões sociais e educacionais, é crucial para manter o nível elevado das publicações.

De modo geral a qualidade editorial da revistas é elevada, porém existem ainda muitos aspectos em que se pode aprimorá-la. Um dos aspectos apontados aqui é uma definição mais precisa do Comitê Editorial, que recebe diferentes nomes (e pode ter diferentes funções). Além disso, uma abertura dos Comitês para a participação de membros de outras instituições é recomendável.

A definição dos conceitos de artigo e relato de experiência também é obscura, e poucas vezes é contemplada nas Diretrizes para Autores ou documento equivalente. Por outro lado, há uma grande predominância de trabalhos publicados cujo conteúdo se refere a relatos de experiências de projetos de extensão. Estas publicações são importantes, porém se revela uma lacuna de abordagens investigativas focadas na extensão universitária, ou em temas que possam subsidiá-la.

A periodicidade das revistas está aquém da desejável, o que pode refletir dificuldades estruturais ou pouca afluência de material publicável de qualidade.

\section{Agradecimentos}

À Gabriela Cordeiro de Oliveira Squariz e à Rubiana do Nascimento Souza pelo auxílio na revisão do texto. Ao FORPROEX - Fórum de Pró-Reitores de Extensão das Instituições Públicas de Educação Superior Brasileiras pelo apoio às atividades editorais e fornecimento de informações.

\section{Notas}

1. No Brasil, a revista Extensão Rural (UFSM) é o veículo mais antigo (1983) dedicado ao campo da extensão, porém em um caráter relativamente disciplinar, voltada aos temas agronômicos e rurais. Deste modo, não foi considerada na presente análise. Antes dela, existiu a Revista Brasileira de Extensão Rural (EMBRATER), que iniciou em 1980, porém encerrou suas atividades na década seguinte.

2. Foram considerados apenas periódicos dedicados exclusivamente à extensão universitária e de caráter multidisciplinar; periódicos com escopo mais amplo (por exemplo, abertos a temas de Ensino, Pesquisa e Extensão) não foram incluídos. Por outro lado, não foram incluídos também periódicos restritos ao tema da Extensão Rural. Para a localização dos periódicos foram utilizadas buscas em bases de indexação (LATINDEX, SEER/IBICT, eLivre, SUMARIO.ORG) e consulta aos pró-reitores de extensão ligados ao FORPROEX - Fórum de Pró-Reitores de Extensão das Instituições Públicas de Educação Superior Brasileiras. Não se trata de uma pesquisa exaustiva, e outras revistas incluídas pelo critério de seleção podem existir e não foram localizadas, especialmente as de caráter impresso, sem divulgação eletrônica.

3. Estão fora desta lista periódicos dedicados à extensão rural agronômica, tais como o Journal of Agricultural Extension and Rural Development (Nigéria), Agricultural Extension \& Rural Development (Canadá), Journal of International Agricultural and Extension Education (Estados Unidos), International Journal of Agricultural Extension (Paquistão), entre outros. Adicionalmente, deve-se ressaltar que a Revista Identidad y Territorio possui um caráter híbrido, mesclando artigos com informes jornalísticos. Outras revistas com caráter predominantemente jornalístico, tais como boletins institucionais, não foram incluídas nesta análise, tanto em nível internacional como nacional.

4. Foram considerados inativos os periódicos que não publicaram nenhuma edição em 2014.

5. Para as revistas mais antigas, considerou-se a periodicidade efetiva, em que pese eventual informação diferente na revista. Para as revistas novíssimas (iniciadas em 2013-2014), considerou-se a periodicidade informada.

6. Foram consideradas apenas revistas em atividade, e iniciadas antes de 2011. 


\section{Referências}

BROWN, H. History and the learned journal. Journal of the History of Ideas, v. 33, n. 3, p. 365-378, 1972.

COELHO, G. C. O papel pedagógico da Extensão Universitária. Em Extensão, v. 13, n. 2, 2014.

JINHA, A. E. Article 50 million: an estimate of the number of scholarly articles in existence. Learned Publishing, v. 23, n. 3, p. 258-263, 2010.

KRZYZANOWSKI, R. F.; FERREIRA, M. C. G. Avaliação de periódicos científicos e técnicos brasileiros. Ciência da Informação, v. 27, n. 2, p. 165-175, 1998.

LIMA, J. L. B.; MIRANDA, A. C. D. Periódicos eletrônicos com o uso do SEER no Rio Grande do Sul: critérios de qualidade. Perspectivas em Gestão \& Conhecimento, v. 1, n. 1, p. 163-179, 2011.

SciELO - Scientific Eletronic Library Online. Critérios, política e procedimentos para a admissão e a permanência de periódicos científicos na Coleção SciELO Brasil. São Paulo, 2014. Disponível em < http://www.scielo.br/avaliacao/ 20141003NovosCriterios SciELO Brasil.pdf > Acesso em 05 mar. 2015.

SOUSA, A. L. A história da extensão universitária. Campinas: Alínea Editora, 2010.

WIKIPEDIA. Philosophical Transactions of the Royal Society. Disponível em: < http://en.wikipedia.org/wiki/ Philosophical_Transactions_of_the_Royal_Society\#Current_pu blication >. Acesso em 04 mar. 2015.

\section{$* * *$}

Como citar este artigo:

COELHO, G. C. Revistas acadêmicas de extensão universitária no Brasil. Revista Brasileira de Extensão Universitária, v. 5, n. 2, p. 69-75, 2014. Disponível em: $<$ https://periodicos.uffs.edu.br/index.php/RBEU/article/view/19 $\underline{43 / \mathrm{pdf}}>$ 